\title{
ACTIVIDADES DE VIGILANCIA EPIDEMIOLÓGICA DE LA MALARIA EN LA RED DIAGNÓSTICA DE LA FRONTERA COLOMBIANA-PERUANA
}

\author{
Mónica Rondón-Cotacio ${ }^{1, a}$, Alberto Tobón-Castaño 2,b
}

\begin{abstract}
RESUMEN
Objetivo. Describir las actividades de la red de diagnóstico de malaria y su control de calidad en la frontera colombianaperuana para caracterizar el funcionamiento del sistema de vigilancia epidemiológica. Materiales y métodos. Estudio descriptivo con enfoque de investigación en implementación. Se visitaron comunidades fronterizas con puestos de atención en salud o voluntarios entrenados para el diagnóstico de malaria. Se georreferenciaron los sitios visitados para conocer su distribución; con información de laboratorios de salud pública departamentales, se analizó el componente de calidad y se evidenció el cumplimiento de los lineamientos nacionales para las redes de diagnóstico de la malaria en la zona de frontera de los dos países. Resultados. Se visitaron 25 sitios colombianos y 18 peruanos, de los cuales, en el $25 \%$ sólo había puestos de microscopía, el $56 \%$ realiza diagnóstico para malaria; y 19\% realiza sólo toma de muestra y las láminas son enviadas a un punto de referencia para su diagnóstico. La Secretaria de Salud del Amazonas (Colombia) no desarrolló visitas de supervisión directa a la red de diagnóstico para malaria en 2016-2017, la Dirección Regional de Salud de Loreto (Perú) no lleva a cabo visitas desde el 2015. El 60\% de los puntos de diagnóstico en el Amazonas y el $29 \%$ en Loreto participaron del aseguramiento de la calidad. Conclusión. Se identificó falta de oportunidad en el diagnóstico en el $44 \%$ de los sitios visitados y deficiencias en componente de calidad de la red de diagnóstico fronteriza.
\end{abstract}

Palabras clave: Monitoreo epidemiológico; Investigación en Servicios de Salud; Malaria; Colombia; Perú (fuente: DeCS BIREME).

\section{EPIDEMIOLOGICAL SURVEILLANCE ACTIVITIES FOR MALARIA IN THE COLOMBIAN-PERUVIAN BORDER DIAGNOSTIC NETWORK}

\begin{abstract}
Objective. To describe the activities of the malaria diagnostic network and its quality control in the Colombian-Peruvian border to characterize the functioning of the epidemiological surveillance system. Materials and Methods. Descriptive study with a research focus on implementation. We visited border communities with health care centers or volunteers trained to diagnose malaria. The visited sites were georeferenced to know their distribution. With information from departmental public health laboratories, the quality component was analyzed and compliance with the national guidelines for malaria diagnostic networks in the border area of the two countries was evidenced. Results. Twenty-five Colombian and 18 Peruvian sites were visited, of which $25 \%$ had only microscopy posts, $56 \%$ made a diagnosis for malaria, and $19 \%$ only took samples and the plates were sent to a reference point for diagnosis. The Ministry of Health of the Amazon (Colombia) did not carry out direct supervision visits to the malaria diagnostic network during 2016-2017; the Regional Health Directorate of Loreto (Peru) has not carried out visits since 2015. Sixty percent of the diagnosis points in the Amazon and $29 \%$ in Loreto participated in quality assurance. Conclusions. Lack of opportunity in the diagnosis was identified in $44 \%$ of the visited sites and deficiencies in the quality component of the border diagnostic network.
\end{abstract}

Keywords: Epidemiological monitoring; Health services research; Malaria; Colombia; Perú (source: MeSH NLM).

\footnotetext{
Facultad Nacional Salud Pública, Universidad de Antioquia. Antioquia, Colombia.

Universidad de Antioquia. Antioquia, Colombia.

Enfermera, magíster en Epidemiología; ${ }^{\text {b }}$ médico, doctor en Salud Pública y Metodología de Investigación Biomédica.

Recibido: 28/03/2018 Aprobado: 22/08/2018 En línea: 28/09/2018
}

Citar como: Rondón-Cotacio M, Tobón-Castaño A. Actividades de vigilancia epidemiológica de la malaria en la red diagnóstica de la frontera colombianaperuana. Rev Peru Med Exp Salud Publica. 2018;35(3):373-81. doi: 10.17843/rpmesp.2018.353.3575. 


\section{INTRODUCCIÓN}

La malaria es un problema de salud pública que afecta a más de 216 millones de personas en 91 países ${ }^{(1)}$. Se transmite en la mayoría de los casos por la picadura de mosquitos hembra del género Anopheles y la intensidad de su transmisión se asocia con factores sociodemográficos y ambientales que en zonas de frontera puede adquirir dimensiones particulares (1); como por ejemplo, las situaciones de conflicto social que propician la migración poblacional y el deterioro del ecosistema, sumado a la falta de coordinación interfronteriza de los programas nacionales de control ${ }^{(2)}$. En tal sentido, la vigilancia de la malaria en zona fronteriza es una tarea de las autoridades en salud para implementar acciones que permitan el mejoramiento de la salud pública y disminución de la carga de enfermedad.

En Colombia y Perú, al igual que en muchos países endémicos, la malaria representa un problema prioritario en salud pública, debido a la tendencia creciente de los casos en determinadas regiones, con un comportamiento cíclico-epidémico que se presenta cada dos a siete años. Así en el Perú, la malaria es endémica en algunas áreas, especialmente en la selva, como lo es el departamento de Loreto ${ }^{(3,4)}$.

En la frontera colombiana-peruana, específicamente entre los departamentos de Amazonas (Colombia) y Loreto (Perú), la vigilancia de la malaria representa un reto, por las limitaciones en la comunicación para realizar la notificación de casos que se han identificado plenamente, a través de, los sistemas de vigilancia epidemiológica. Hasta la semana epidemiológica 52 de 2016 se notificaron al SIVIGILA colombiano 83356 casos de malaria de los cuales el $4,2 \%$ fueron aportados por el departamento del Amazonas ${ }^{(5)}$. Del mismo modo en Perú, durante el 2016, se notificaron 55964 casos de malaria y Loreto concentró el $95,6 \%$ de los casos ${ }^{(6)}$.

Ante ello, los casos de malaria en Colombia o Perú, posiblemente no reciben un tratamiento, seguimiento y evaluación de la manera que lo ordenan las normas de cada país. Esta desarticulación posibilita que se presente un subregistro de casos o notificación incompleta en los sistemas de vigilancia epidemiológica lo cual tendrá repercusiones en las actividades de control de la malaria especialmente en zona fronterizas. Además, se debe tener en cuenta que el componente de detección de casos, es considerado uno de los elementos esenciales de los programas para el control de malaria y eje para la realización de las actividades de vigilancia epidemiológica ${ }^{(7,8)}$.

Las zonas de frontera endémicas para malaria pueden ofrecer limitaciones en la aplicación de los programas de salud y las acciones de control de las enfermedades debido

\section{MENSAJES CLAVE}

Motivación para realizar el estudio. La vigilancia epidemiológica constituye un pilar esencial para el control de la malaria. La Organización Mundial de la Salud estimó que el 2015 los sistemas de vigilancia de malaria informaron sólo el 19\% de casos presentados mundialmente y que no se realiza la totalidad de diagnósticos esperados.

Principales hallazgos. Existe una falta de oportunidad en el diagnóstico de la malaria y no se está realizando supervisión a los puntos de diagnóstico en la zona de frontera colombiana-peruana.

Implicancias. El subregistro y/o el diagnóstico presuntivo de casos generan incoherencia entre la situación real de malaria y acciones de atención y control, llevando a falta de efectividad de los programas, aumento en magnitud del problema y uso inadecuado de recursos.

a sus características propias como lo son la dispersión geográfica, la dificultad para control de la migración fronteriza, la presencia de actividades ilegales, entre otras ${ }^{(7)}$. Así, un estudio con el enfoque de investigación en implementación (implementation research) podría aproximarse a establecer cuáles son las dificultades y barreras que enfrentan los programas de salud para aplicar intervenciones y desarrollar estrategias en zonas fronterizas ${ }^{(9)}$.

El objetivo del presente estudio es describir las actividades de la red de diagnóstico de la malaria y su control de calidad en zona de frontera colombiana-peruana, con el fin de brindar información a los tomadores de decisiones, para el mejoramiento del sistema de vigilancia epidemiológica existente.

\section{MATERIALES Y MÉTODOS}

\section{TIPO DE ESTUDIO}

Estudio descriptivo con enfoque de métodos mixtos (combinación de métodos cuantitativos y métodos cualitativos), convergente paralelo ${ }^{(10)} \mathrm{e}$ investigación de implementación. El componente cuantitativo se desarrolló a través de una encuesta y el componente cualitativo a través de una entrevista semiestructurada ${ }^{(11)}$.

\section{ÁREA DE ESTUDIO Y SELECCIÓN DE PARTICIPANTES}

El componente cuantitativo del estudio se realizó en localidades de la frontera colombiana-peruana; departamentos de Amazonas en Colombia y Loreto en Perú, en las zonas aledañas a los ríos Putumayo y Amazonas. Mediante un muestreo por conveniencia, se seleccionaron y visitaron 43 comunidades, 25 colombianas (Anexo 1) y 18 peruanas (Anexo 2), durante los meses de abril a junio de 2017, considerando la accesibilidad geográfica y presencia de 
casos de malaria (evidenciado a través de la notificación a los sistemas de vigilancia de cada país) como principal criterio de elegibilidad. Para el componente cualitativo, se indagó con los responsables de los programas de Malaria, Epidemiología y Laboratorio de Salud Pública Departamental (LSPD) acerca de sus percepciones sobre el funcionamiento de la estrategia de vigilancia epidemiológica para la malaria en la zona de frontera.

\section{VARIABLES}

Las variables incluidas en el estudio fueron: a) Cobertura geográfica: definida como la ubicación espacial de las comunidades visitadas; y b) Calidad del diagnóstico: entendida como la concordancia en las últimas evaluaciones externas directas del desempeño, realizadas por el LSPD a su red de diagnóstico.

Asimismo, mediante listas de chequeo o aplicación de cuestionario con preguntas cerradas, se recogieron variables como nacionalidad de la población atendida, número de comunidades atendidas, tiempo en minutos de la comunidad más alejada, tipo de diagnóstico que realiza el punto visitado, si poseía microscopio exclusivo para malaria, y se obtuvo información del número de puntos de diagnóstico y su funcionamiento. Finalmente, se elaboró un mapeo básico de la ubicación de estos puntos visitados (mediante georreferenciación de los puntos de diagnóstico).

\section{ANÁLISIS DE DATOS}

Se construyó una base de datos con la información obtenida en la recolección de la información cuantitativa. Los cuestionarios se identificaron con un código y se realizó control de calidad por una segunda persona. Se realizó un análisis descriptivo con frecuencias absolutas y porcentuales; asimismo, se presentan medianas y su rango intercuartil (RIQ) para las variables sin distribución normal.

Se geocodificaron los datos espaciales para determinar la distribución de los puntos visitados y mostrar los recorridos realizados en el desarrollo del trabajo. Los datos recopilados fueron examinados y analizados en los programas estadísticos Stata $® 14$ (StataCorp LP, Texas, Estados Unidos), Epi-info ${ }^{\circledR}$ y ArcGIS $®$ 10.5, licenciados para la Universidad de Antioquia.

Se buscó evidenciar la calidad de la red de diagnóstico revisando el reporte de los puntos visitados, entregados en la evaluación externa del desempeño (EED) por parte de los LSPD y la concordancia del diagnóstico. Contrastando estos resultados con fuentes secundarias entregados por los laboratorios de salud pública o de referencia de cada departamento fronterizo, informes de visita de supervisión realizados por los LSPD y EED. En la EED se solicitan láminas a los microscopistas para evaluar la concordancia del diagnóstico, concordancia en el recuento y establecer errores técnicos.

Además, se realizaron entrevistas semiestructuradas apoyadas de una guía a los responsables de los programas de Epidemiología, Malaria y LSPD de los departamentos de Amazonas y Loreto, con la finalidad de evidenciar las percepciones de los responsables sobre el funcionamiento del sistema de vigilancia epidemiológico para este evento.

Para el análisis mixto, mediante una previa categorización de los resultados y un análisis de contenido, se evidenciaron los resultados cuantitativos, complementados con la información cualitativa para de esta manera establecer si existió o no convergencia con la información reportada.

\section{CONSIDERACIONES ÉTICAS}

El estudio fue aprobado por el Comité de Bioética de la Sede Investigación Universitaria de la Universidad de Antioquia, y contó con autorizaciones de la Secretaria de Salud del Amazonas (SSA) y Dirección Regional de Salud de Loreto. En Colombia, se contó con avales de autoridades indígenas tradicionales, y en los dos países de la firma de consentimiento informado por cada uno de los participantes antes de aplicar el cuestionario y de realizar las entrevistas.

\section{RESULTADOS}

Se realizaron dos recorridos fluviales (Figura 1). Primero por la cuenca del río Putumayo visitando 25 comunidades: 15 colombianas y diez peruanas; el segundo sobre la cuenca del río Amazonas, se visitaron 18 comunidades: diez colombianas y ocho peruanas (Figura 2).

La proporción de sitios visitados que contaban con un punto de diagnóstico o de microscopía fue del 56\% (24/43), con funcionamiento intermitente, de acuerdo a la disponibilidad del recurso humano, de los insumos y de los equipos. La atención de los pacientes con malaria (diagnóstico, tratamiento y seguimiento) se encontró enmarcada en el sistema de salud de cada país. De los 43 puntos visitados (Anexo 1 y 2), 11 (25\%) correspondieron a puestos de microscopía, nueve del departamento de Amazonas y dos de la región de Loreto.

Por el río Putumayo los puntos de diagnóstico están concentrados estratégicamente, cercanos a centros poblados de mayor tamaño. La mitad de los sitios visitados atienden por lo menos cuatro comunidades, sin importar la nacionalidad. El tiempo para realizar el recorrido para acceder a la atención desde comunidades diferentes a la 


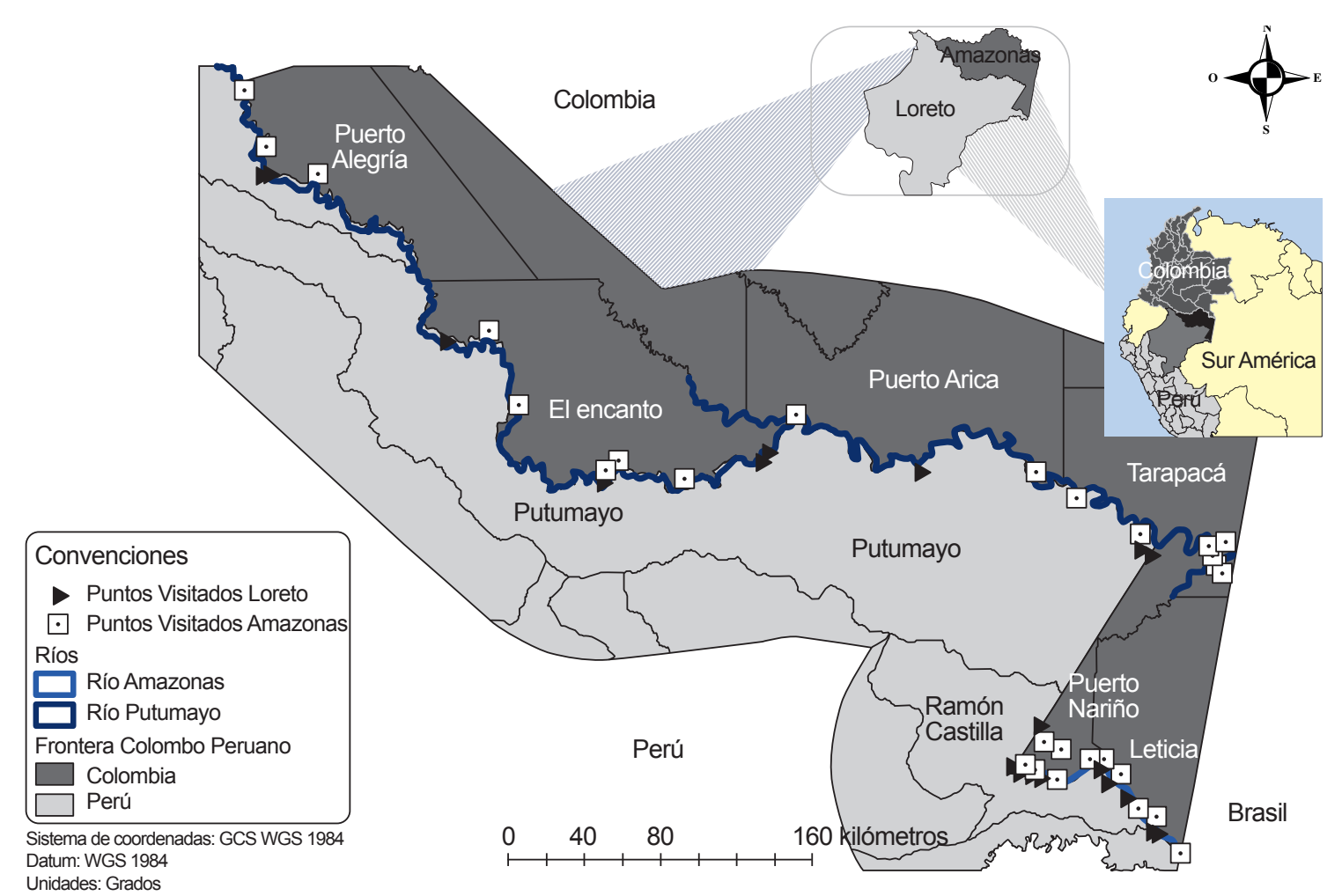

Figura 1. Ubicación de sitios visitados según recorridos fluviales en la frontera colombiana-peruana.

visitadas puede ser de hasta 24 horas. Dichas distancias fueron medidas en minutos, tomando como base el recorrido por río en un «peque-peque» (vehículo tradicional usado como medio de transporte en los ríos selváticos, similar a una canoa motorizada) y de acuerdo a la experiencia de las personas de la zona. La mayoría de las comunidades en esta zona, se encuentran a la ribera de un río principal o su afluente.
El 56\% (24/43) de los puntos visitados se clasificaron como puestos básicos de salud, los cuales contaban con un promotor de salud en el caso peruano o con un auxiliar en salud pública en el caso colombiano. El 46\% (20/43) de los puntos visitados reportaron realizar todas las actividades indagadas y el $19 \%$ sólo la toma de muestra, es decir, elaboran la gota gruesa y el extendido de sangre, y envían las láminas a su punto de referencia más cercano para que se realice la lectura (Tabla 1).

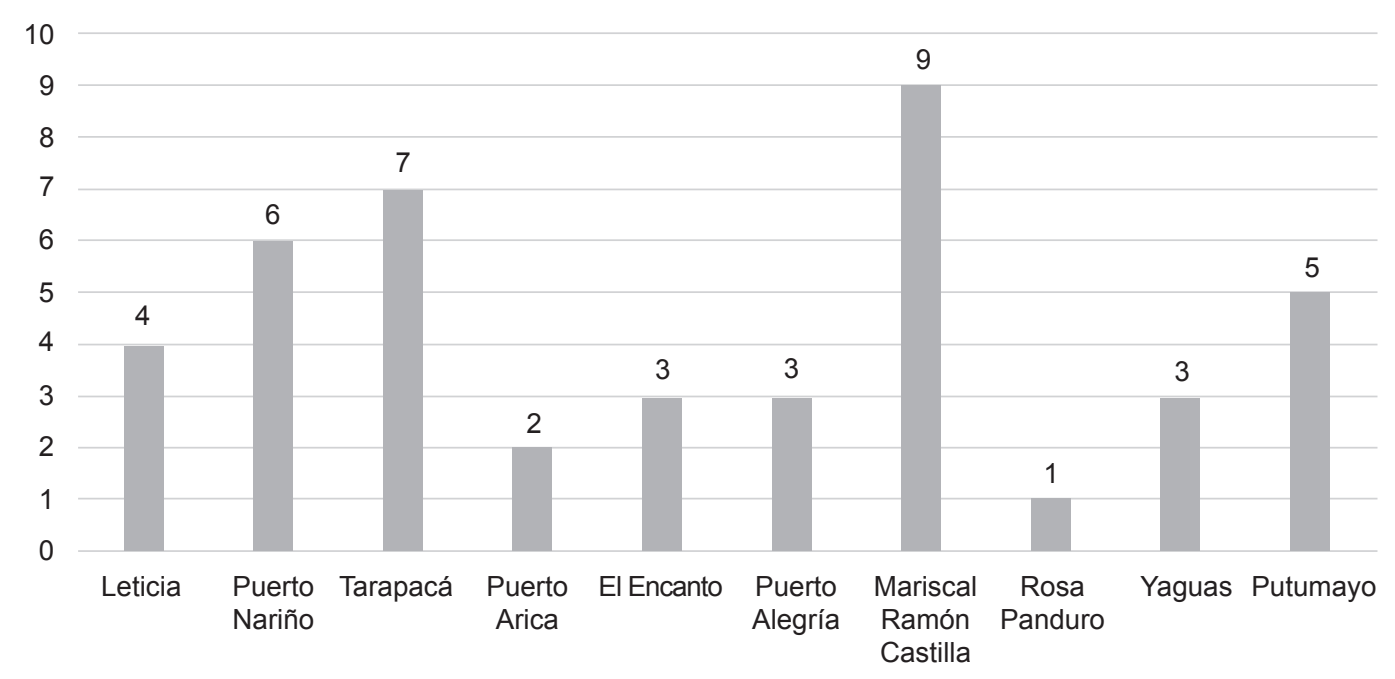

Figura 2. Número de comunidades visitadas según localidades de la frontera colombiana-peruana. 
Tabla 1. Características de los puntos de salud visitados por departamento en la zona de frontera colombiana-peruana.

\begin{tabular}{|c|c|c|c|c|}
\hline \multirow{2}{*}{ Característica } & \multicolumn{2}{|c|}{ Amazonas $(n=25)$} & \multicolumn{2}{|c|}{ Loreto $(n=18)$} \\
\hline & $\mathrm{n}$ & $\%$ & $\mathrm{n}$ & $\%$ \\
\hline \multicolumn{5}{|l|}{ Tipo de punto diagnóstico visitado } \\
\hline Laboratorio clínico en centro de atención de salud & 2 & 8,0 & 3 & 16,7 \\
\hline Puesto microscopia dentro de centro de atención de salud & 0 & 0,0 & 3 & 16,7 \\
\hline Punto de microscopía & 9 & 36,0 & 2 & 11,1 \\
\hline Otro* & 14 & 56,0 & 10 & 55,6 \\
\hline \multicolumn{5}{|l|}{ Servicios que presta** } \\
\hline Sólo toma de muestra & 4 & 16,0 & 4 & 22,2 \\
\hline Diagnóstico & 10 & 40,0 & 14 & 77,8 \\
\hline Prescribe tratamiento & 12 & 48,0 & 14 & 77,8 \\
\hline Entrega tratamiento & 13 & 52,0 & 14 & 77,8 \\
\hline Seguimiento & 18 & 72,0 & 12 & 66,7 \\
\hline Vigilancia & 22 & 88,0 & 14 & 77,8 \\
\hline Presta todos los servicios $\pi$ & 10 & 40,0 & 10 & 55,6 \\
\hline \multicolumn{5}{|l|}{ Nacionalidad de población atendida } \\
\hline Colombiana & 10 & 40,0 & 0 & 0,0 \\
\hline Peruana & 0 & 0,0 & 3 & 16,7 \\
\hline Brasileña & 1 & 4,0 & 1 & 5,6 \\
\hline Ambos (Col-Per) & 14 & 56,0 & 14 & 77,8 \\
\hline
\end{tabular}

* Punto de referencia de salud básico con un promotor en salud (Perú) o un auxiliar en salud pública (Colombia).

* Un punto de salud visitado puede prestar más de un servicio.

$\pi$ Incluye: Toma de muestra, diagnóstico, prescribe y entrega tratamiento, seguimiento y vigilancia.

Col: Colombia; Per: Perú.

Laboratorio clínico: realiza otro tipo de pruebas, además de la gota gruesa o de la prueba de diagnóstico rápido y se encuentra dentro de un centro de atención de salud. Puesto microscopia dentro de centro de atención de salud: realiza sólo gota gruesa o prueba de diagnóstico rápido y se encuentra dentro de centro de atención de salud. Punto de microscopia: realiza sólo gota gruesa o prueba de diagnóstico rápido y se ubica por fuera de una institución de salud (son muy comunes en Colombia). La mayoría funciona en la vivienda del microscopista.

De acuerdo a lo manifestado por los promotores de salud en Perú, la mayoría de los casos que reciben tratamiento antimalárico tiene un diagnóstico presuntivo, ya que los resultados de la gota gruesa pueden tardar días en ser informados a los puntos de salud por las distancias y dificultades en las comunicaciones existentes. La malaria en ambos países es notificada a los sistemas de vigilancia epidemiológicos como casos confirmados y fue informado de la siguiente manera:

"...Y para que un puesto de salud remita las muestras a un lugar $y$ den el tratamiento es otra dificultad que tenemos. Entonces hay un problema de oportunidad del diagnóstico que es un tema este muy importante...» (Funcionario/a DIRESA-Loreto)

El 65\% (28/43) de los puntos visitados atienden personas de ambos países, predominando esta situación en el lado peruano (77\%). Frente a lo cual un participante afirmó:

«... por ejemplo, que si hay una situación ya que es una urgencia que pone en riesgo la vida de la persona, entonces que tanto el vecino... fronterizo o... lo atienda para que digamos no vaya a suceder una mortalidad...esas urgencias vitales por ejemplo se tratan...» (Funcionario/a de la Secretaría de Salud de Amazonas)

Las redes de diagnóstico para malaria de los departamentos de Amazonas y Loreto son lideradas por sus respectivos
LSPD; dicha red, ejecuta sus acciones teniendo en cuenta los lineamientos entregados por la Red Nacional de Laboratorios (RNL) que lidera el Instituto Nacional de Salud de cada país.

De acuerdo a la SSA, durante el 2016 y hasta el 30 de junio de 2017, no se desarrollaron visitas de supervisión directa a la red de diagnóstico para malaria. Para la dirección regional de salud de Loreto (DIRESA-Loreto), estas visitas no se llevan a cabo desde el 2015. Al respecto se mencionó:

«...pero lo que nos está faltando es verificar, ir y verificar si realmente estos materiales los están usando, si... han sido distribuidos, porque la distribución la hacemos a nuestros laboratorios supervisores....» (Funcionario/a DIRESA-Loreto)

Para 2016 y 2017 la red de microscopía en la zona de frontera en el departamento del Amazonas, estaba conformada por siete puestos, de los cuales tres (43\%) participaron en la EED. En el caso de Perú, la red de diagnóstico en la frontera, para el mismo período de tiempo, contó con 13 puntos distribuidos entre puestos y centros de salud; de estos, cinco puntos en el 2016 y 2017 (38\%) participaron en la EED. Lo reportado por los sitios visitados se presenta en la Tabla 2. 
Tabla 2. Componente de calidad del diagnóstico reportado por los sitios visitados que realizan diagnóstico en la frontera colombiana-peruana.

\begin{tabular}{|c|c|c|c|c|c|c|}
\hline \multirow{2}{*}{ Característica } & \multicolumn{2}{|c|}{ Amazonas $(n=10)$} & \multicolumn{2}{|c|}{ Loreto $(n=14)$} & \multicolumn{2}{|c|}{ Total } \\
\hline & $\mathbf{n}$ & $\%$ & $\mathbf{n}$ & $\%$ & $\mathbf{n}$ & $\%$ \\
\hline \multicolumn{7}{|l|}{ Tipo de diagnóstico realizado por punto visitado } \\
\hline Sólo gota gruesa (GG) & 9 & 90,0 & 6 & 42,9 & 15 & 62,5 \\
\hline Sólo prueba de diagnóstico rápido (PDR) & 0 & 0 & 6 & 42,9 & 6 & 25,0 \\
\hline Ambos & 1 & 10,0 & 2 & 14,3 & 3 & 12,5 \\
\hline Cita al paciente para seguimiento & 9 & 90,0 & 14 & 100 & 23 & 95,8 \\
\hline \multicolumn{7}{|l|}{ Número de veces que se cita $(n=23)^{*}$} \\
\hline Una & 2 & 22,2 & 5 & 35,7 & 7 & 30,4 \\
\hline Dos & 4 & 44,4 & 4 & 28,6 & 8 & 34,8 \\
\hline Tres & 3 & 33,3 & 5 & 35,7 & 8 & 34,8 \\
\hline Toma muestra por demanda & 8 & 80,0 & 11 & 78,6 & 19 & 79,2 \\
\hline Realiza búsqueda activa del paciente & 7 & 70,0 & 9 & 64,3 & 16 & 66,7 \\
\hline Participa en el aseguramiento de la calidad & 8 & 80,0 & 7 & 50,0 & 15 & 62,5 \\
\hline Recibió supervisión directa en los últimos seis meses & 5 & 50,0 & 1 & 7,1 & 6 & 25,0 \\
\hline
\end{tabular}

* Con respecto a los que contestaron que citaban el paciente a seguimiento.

La concordancia entre los diagnósticos realizados en los distintos puntos y el LSPD del Amazonas presentó un promedio en el 2016 y 2017 de $54,2 \%$ y $26,7 \%$ respectivamente. Para el departamento de Loreto fue de $71 \%$ en el 2016 frente $89 \%$ en el 2017 . El patrón de referencia por los niveles nacionales es más del $95 \%$.

El $75 \%$ de los puntos visitados realizan diagnóstico a través de gota gruesa y de estos, el $56 \%$ deben compartir el uso del microscopio con el programa de tuberculosis, o con la lectura de otro tipo de muestras cuando está ubicado en laboratorios clínicos. Amazonas no tiene implementado el uso de la PDR (Tabla 2).

En el componente de recurso humano que realiza el diagnóstico, en Perú, se encontró que el $57 \%$ de los puestos visitados no realizan diagnóstico por gota gruesa y aunque complementan su diagnóstico a través de las PDR, la disponibilidad de este insumo no es constante.

En Amazonas, la capacidad de la red de microscopía estuvo garantizada con actividades anuales de entrenamiento y actualización, por este motivo los microscopistas gozan de buena reputación y confianza de su desempeño entre los tomadores de decisiones. Sobre esto se relató:

«Nosotros hacemos una inversión anual de 20 millones de pesos (aproximadamente USD 6900) en coordinación con la jefe de promotores los haga llegar a Leticia, los recapacitamos, los reentrenamos en materia de notificación, pero ya es inherente al laboratorio de salud pública sobre el tema de diagnóstico y demás...» (Funcionario/a de la Secretaría de Salud de Amazonas)

Al indagar la disponibilidad de tiempo para la atención del puesto de salud en la comunidad, se pudo evidenciar que el número de días laborables, para Amazonas y Loreto, tuvo una mediana de cinco y seis días (RIQ 3,5-7 y 5-7) respectivamente, y el número de horas laborables al día tuvo una mediana ocho y seis horas (RIQ 6-9 y 6-8,5) respectivamente; dicha información coincidió con lo que se pudo evidenciar en terreno, en donde la mayoría del personal que presta el servicio de diagnóstico de malaria es propio de la zona, la cual conserva sus costumbres y raíces, e intercalan su actividad en salud con actividades como la caza y la pesca.

Con respecto a la distribución de las incidencias parasitarias anuales (IPA), la mayoría de las localidades fronterizas, se encuentran en riesgo alto (IPA 10 a 49,99 x 1000 habitantes) y sólo uno de ellos está en riesgo muy alto (Tarapacá IPA $>50 \times 1000$ habitantes) (Figura 3).

\section{DISCUSIÓN}

De las 43 comunidades visitadas en la frontera colombianaperuana, un $25 \%$ contaban con puestos de microscopía. En el $56 \%$ se realiza diagnóstico para malaria; $19 \%$ realiza sólo toma de muestra y las láminas son enviadas a punto de referencia para diagnóstico. La SSA no desarrolló visitas de supervisión directa a la red diagnóstica para malaria en 20162017, la DIRESA-Loreto no realiza visitas desde el 2015. El $60 \%$ de los puntos de diagnóstico en el Amazonas y el $29 \%$ en Loreto participaron del aseguramiento de la calidad.

La vigilancia en salud pública es uno de los pilares en la Estrategia Técnica Mundial contra la malaria 2016-2030 propuestos por la Organización Mundial de la Salud (OMS); las deficiencias en esta función esencial de la salud 


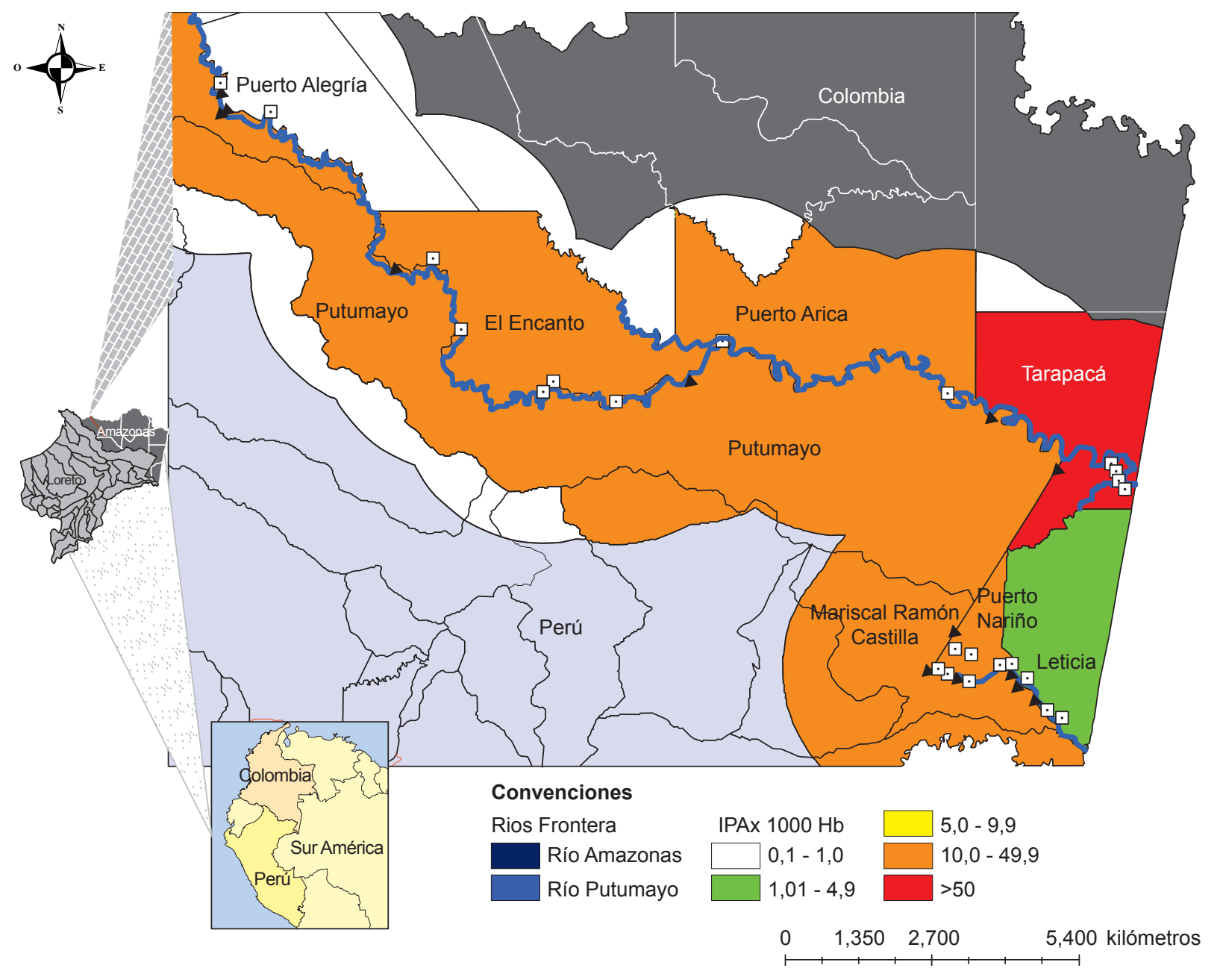

Figura 3. Incidencias Parasitarias Anuales (IPA) por localidades fronterizas, 2017.

Fuente: SIVIGILA-RENACE 2017 Departamento Administrativo Nacional de Estadística (DANE); Instituto Nacional de Estadística e Informática (INEI).

pública han sido identificadas como uno de los problemas que dificulta la óptima intervención para el control de esta enfermedad ${ }^{(12)}$, y que suele ocurrir en las poblaciones con acceso geográfico difícil, como es el caso de las que se encuentran en la frontera entre Colombia y Perú, en los departamentos de Amazonas y Loreto. En la actualidad, existen sistemas de vigilancia epidemiológica en los países de Colombia y Perú, cuyos objetivos están encaminados a proveer información sistemática y oportuna que apoyen la toma de decisiones en salud pública ${ }^{(13)}$.

Uno de los retos identificados por la OMS en las zonas de baja transmisión de malaria, como lo es la zona de estudio, es la necesidad de transformar la vigilancia epidemiológica en una intervención regular, con el fin de que se obtengan beneficios adicionales a los esperados ${ }^{(12)}$. Sin embargo, una revisión sistemática realizada en el 2015 reporta que en siete países africanos y asiáticos, los sistemas de vigilancia epidemiológica están funcionando actualmente en las zonas de frontera sin integrarse a las dinámicas de los sistemas de salud existentes ${ }^{(14)}$. En temas de atención de salud, es responsabilidad de los sistemas de cada país garantizar de manera integral, la atención de toda la población, teniendo en cuenta la situación de sus pobladores, las dificultades que presentan para acceder a los servicios de salud, como los tiempos y costos implicados en la búsqueda de la atención y derivados de esta (compra de medicamentos, ayudas diagnósticas, etc.). Por ejemplo, las personas que viven en la frontera colombiana-peruana, se desplazan en canoas de madera (peque-peque), lo que hace que el recorrido entre comunidades para buscar atención en salud sea prolongado y costoso ${ }^{(15)}$.

Aunque en Colombia y Perú la cobertura del sistema de salud se considera casi universal para la población de menos recursos, las dificultades que se presentan para el acceso (geográficas, financieras y de carácter administrativo) ${ }^{(16,17)}$, se configuran con más fuerza en las zonas de frontera, ocasionando que la atención para los pacientes se realice sin continuidad y eficiencia por parte 
de los responsables directos de la prestación de servicios de salud ${ }^{(15)}$.

En este estudio se evidenció una oferta deficitaria de los servicios, pues a pesar de existir una cantidad de puestos de salud, centros de salud, hospitales y/o puestos de microscopía ubicados de forma estratégica, muchos de ellos carecen de elementos necesarios para la atención del paciente con malaria, como microscopios, insumos para toma de muestra y coloración, recurso humano entrenado para la lectura, etc. Por ejemplo, en algunas comunidades del Perú, se realiza el diagnóstico presuntivo de casos, generalmente en aquellos lugares en donde no se realiza el diagnóstico con gota gruesa y cuando no se cuentan con PDR ${ }^{(18)}$. Situación similar pudo ser evidenciada en Nigeria, en un estudio donde se identificaron los principales cuellos de botella por las partes interesadas para la implementación operativa de las políticas de atención de la malaria ${ }^{\left({ }^{8)}\right.}$. No obstante, una fortaleza de la oferta es la atención abierta y sin barreras a la población fronteriza, como factor común a ambos lados de la misma.

El componente de la calidad, busca mantener óptimos los estándares normados para un diagnóstico confiable, veraz y oportuno. Se evalúa a través de las redes de diagnóstico por parte de los LSPD en el nivel departamental o regional, quienes a su vez son evaluados por las redes nacionales de laboratorio de cada país ${ }^{(19,20)}$. En ambos países se busca la disponibilidad de recurso humano idóneo y entrenado, insumos, equipos y procesos establecidos para realizar el diagnóstico de la malaria ${ }^{(19)}$. Los hallazgos de este estudio, en el componente de diagnóstico, evidenciaron que en Perú, el $57 \%$ de los puestos visitados no realizan diagnóstico por gota gruesa y aunque complementan su diagnóstico a través de las PDR, la disponibilidad de estas no es permanente. En Colombia, la situación es similar y aunque la mayoría del recurso humano encargado del diagnóstico a través de la gota gruesa se encuentra entrenado para tal fin, sólo el $40 \%$ de los puntos visitados cuentan con un microscopio para esta actividad.

El diagnóstico presuntivo de casos por parte del recurso humano que tiene mayor experiencia para el manejo de casos de malaria, como son los promotores o microscopistas, podría suponer un mayor registro de casos, teniendo en cuenta que en ambos países los casos ingresan al sistema de vigilancia epidemiológico como casos confirmados ${ }^{(2,21)}$. Situación al parecer común en las zonas rurales del Perú (18), en donde sólo el $20 \%$ de los casos que reciben tratamiento presuntivo son verdaderos positivos, es decir, los demás reciben tratamiento sin tener la enfermedad, lo que podría contribuir a aumentar la resistencia a los medicamentos antimaláricos. Tal vez esta situación pueda ser explicada por los hallazgos relacionados con el componente de calidad de la red de diagnóstico, en donde la falta de acompañamiento durante los últimos años a estos puntos de atención y recurso humano, mediante las visitas de supervisión directa incidió en que las redes de laboratorio de cada país no estén fortalecidas.

Dentro de las limitaciones del presente estudio se resalta la no inclusión de la percepción del recurso humano encuestado en campo, sin embargo, no se contempló esto por temas de tiempo y logística. Debido a la dispersión geográfica, tiempo y costos necesarios para su desarrollo, se tuvo como limitación, el no poder visitar todas las comunidades de la zona de frontera, para conocer de una manera más completa la situación del sistema de vigilancia epidemiológica en el corredor fronterizo; quedando sin ser visitadas cuatro comunidades en cada país.

En conclusión, se identificó una oferta deficitaria de los servicios de salud visitados en la frontera colombianaperuana, pues muchos de ellos carecen de elementos necesarios para la atención oportuna del paciente con malaria; sumado a deficiencias en el componente de calidad de la red de diagnóstico. Es necesario que se reorienten los procesos de atención, asistencia técnica y supervisión para que la red de diagnóstico y vigilancia epidemiológica se desarrollen de manera permanente en la zona de frontera.

Agradecimientos: a la Dirección de Salud Regional de Loreto, Secretaria de Salud Departamental del Amazonas, al Programa Especial de Investigación y Entrenamiento en Enfermedades Tropicales (TDR), a la Universidad de Antioquia: grupo de Epidemiología y de Malaria.

Contribuciones de los autores: MRC participó en la elaboración del protocolo, coordinación del estudio en campo, concepción y diseño del estudio, recolección, análisis e interpretación de datos y redacción del artículo. ATC participó en la elaboración del protocolo, concepción y diseño del estudio, análisis e interpretación de datos y redacción del artículo.

Fuentes de financiamiento: este trabajo de investigación fue financiado por la Secretaria de Salud del Amazonas Colombia, el Programa Especial de Investigaciones y Enseñanzas sobre Enfermedades Tropicales (TDR) y la Universidad de Antioquia.

Conflictos de interés: los autores declaran no tener conflictos de interés.

\section{REFERENCIAS BIBLIOGRÁFICAS}

1. World Health Organization. World Malaria Report 2017 [Internet]. Geneva: WHO; 2017 [citado el 23 de enero de
2017 ]. Disponible en: http://apps.who. int/iris/bitstream/10665/259492/1/97 89241565523-eng.pdf?ua $=1$
2. Instituto Nacional de Salud. Protocolo de vigilancia en Salud Pública. Malaria [Internet]. Bogotá: INS; 2014 [citado 
el 23 de enero de 2017]. Disponible en: http://www.ins.gov.co/lineas-de-accion/Subdireccion-Vigilancia/sivigila/ Protocolos SIVIGILA/PRO Malaria. pdf

3. Instituto Nacional de Salud. Boletín Epidemiológico Semanal Semana 52 [Internet]. Bogotá: INS; 2017 [citado el 26 de enero de 2018 ]. Disponible en: http://www.ins.gov.co/buscador-eventos/ BoletinEpidemiologico/2017Boletín epidemiológico semana 52.pdf

4. Ministerio de Salud de Peru. Boletín Epidemiológico del Perú [Internet]. Lima: MINSA; 2017 [citado el 26 de diciembre de 2017]. Disponible en: http://www. dge.gob.pe/portal/docs/vigilancia/ boletines/2017/52.pdf

5. Instituto Nacional de Salud. Boletín Epidemiológico Número 52 [Internet]. Bogotá: INS; 2016 [citado el 1 de diciembrede2017]. Disponibleen:http:// www.ins.gov.co/boletin-epidemiologico/ Boletn Epidemiolgico/2016 Boletín epidemiológico semana 52 -.pdf

6. Centro Nacional de Epidemiología Prevención y Control de Enfermedades. Boletín Epidemiológico del Perú, semana 52 [Internet].Lima: Centro Nacional de Epidemiología prevención y control de enfermedades ; 2016 [citado el 26 de diciembre de 2017]. Disponible en: http://www.dge.gob.pe/portal/docs/ vigilancia/boletines/2016/52.pdf

7. Organismo Andino de Salud. Convenio Hipólito Unanue. Guía andina de vigilancia de casos y brotes para ámbitos de frontera [Internet]. Lima: OAS; 2007 [citado el 5 de mayo de 2017]. Disponible en: http://www.orasconhu.org/documentos/ ult_publi_guia_rave.pdf

8. Onyiah P, Adamu AMY, Afolabi RF, Ajumobi O, Ughasoro MD, Odeyinka O, et al. Bottlenecks, concerns and needs in malaria operational research: The perspectives of key stakeholders in Nigeria. BMC Res Notes. 2018;11:272. doi: https://doi. org/10.1186/s13104-018-3379-5

9. World Health Oganization. Implementation Research Toolkit [Internet]. Geneva: WHO; 2014. [citado el 23 de enero de 2017]. Disponible en: http://www.who. int/tdr/publications/topics/ir-toolkit/en/

10. Creswell J, Plano V. Design and Conducting Mixed Methods Research. 2a ed. SAGE; 2011.

11. Díaz-Bravo L, Torruco-García U, Martínez-Hernández M, Varela-Ruiz M. La entrevista, recurso flexible y dinámico. Investig en Educ Médica. 2013;2(7):162-7.

12. Organización Mundial de la Salud. Estrategia Técnica Mundial Contra La Malaria 2016-2030 [Internet]. Geneva: OMS; 2015 [citado el 9 de noviembre de 2016 ]. Disponible en: http:// www.who.int/malaria/publications/ atoz/9789241564991/es/

13. Colombia, Ministerio de la Protección Social. Decreto Número 3518 de 2006 por el cual se crea y reglamenta el Sistema de Vigilancia en Salud Pública y se dictan otras disposiciones. Bogotá: Ministerio de la Protección Social; 2006.

14. Ohrt C, Gosling RD, Wegbreit J, Lee BY, Roberts KW, Sturrock HJW. Information Systems to Support Surveillance for Malaria Elimination. Am J Trop Med Hyg. 2015;93(1):145-52.

15. Krisher LK, Krisher J, Ambuludi M, Arichabala A, Ayala EB, Navarrete P, et al. Successful malaria elimination in the Ecuador - Peru border region: epidemiology and lessons learned. Malar J. 2016;15(1):573.

16. Ministerio de Salud y Protección Social. Estudio sobre el modo de gestionar la salud en Colombia [Internet]. Bogotá: Minis- terio de Salud y Protección Social; 2012 [citado el 30 de noviembre de 2017]. Disponible en: https://www.minsalud.gov. co/Documentos y Publicaciones/Estudio sobre el modo de gestionar la salud en Colombia.pdf

17. Perú, Congreso de la República. Ley No 26842, Ley General de Salud. Lima: Congreso de la República; 1997.

18. Arróspide N. Tratamiento presuntivo de malaria por promotores de salud en comunidades periurbanas de Iquitos. Rev Peru Med Exp Salud Publica. 2005;22(3):241-2

19. Instituto Nacional de Salud. Manual de procedimientos de laboratorio para el diagnóstico de malaria. Serie de Normas Técnicas No39 [Internet]. Lima: INS; 2003 [citado el 20 de junio 2017]. Disponible en: http://www.ins.gob.pe/ repositorioaps/0/4/jer/-1/Antimalaricos/ manualMALARIA.pdf

20. Instituto Nacional de Salud. Manual para el diagnóstico de malaria no complicada en puestos de diagnóstico y tratamiento [Internet]. Bogotá DC: INS; 2015 [citado el 5 de junio de 2017]. Disponible en: https://www.minsalud.gov.co/sites/ rid/Lists/BibliotecaDigital/RIDE/IA/ INS/manual-diagnostico-malaria-nocomplicada.pdf

21. Ministerio de Salud. Protocolos de Vigilancia Epidemiologica [Internet]. Lima: MINSA; 2006 [citado el 5 de junio de 2017]. Disponible en: http://bvs.minsa.gob.pe/ local/MINSA/1382-1.pdf

Correspondencia: Mónica Rondón Cotacio Dirección: Sede de Investigaciones Universitarias Cra.53 N0.61-30 Lab. 610, Universidad de Antioquia. Medellin, Colombia.

Teléfono: +573132821871

Correo electrónico: moroco861@hotmail.com 


\section{ANEXOS}

Anexo 1. Características sobre el diagnóstico de la malaria en las puntos visitados del departamento de Amazonas (Colombia), 2017.

\begin{tabular}{|c|c|c|c|c|c|}
\hline Puntos visitados & Tipo de recurso humano que labora & $\begin{array}{l}\text { Tienen microscopio } \\
\text { exclusivo para malaria }\end{array}$ & $\begin{array}{l}\text { Tipo de } \\
\text { diagnóstico que } \\
\text { realizan }\end{array}$ & $\begin{array}{l}\text { Número de } \\
\text { comunidades } \\
\text { que atienden }\end{array}$ & $\begin{array}{l}\text { Nacionalidad de } \\
\text { pacientes que } \\
\text { atienden }\end{array}$ \\
\hline Puerto Perea & Microscopista & Sí, pero no exclusivo & GG y PDR & 4 & Col/Per \\
\hline Peña Blanca & Auxiliar en salud pública & No tiene & No realiza & 1 & Colombiana \\
\hline Puerto Alegría & Microscopista & No tiene & No realiza & 4 & Col/Per \\
\hline El Encanto & Auxiliar en salud pública & No tiene & No realiza & 1 & Colombiana \\
\hline Puerto Belén & Microscopista & Sí & GG & 6 & Col/Per \\
\hline Marandúa & Auxiliar en salud pública & No tiene & No realiza & 1 & Colombiana \\
\hline Sabaloyaco & Auxiliar en salud pública & No tiene & No realiza & 1 & Col/Per \\
\hline Cartagena & Auxiliar en salud pública & No tiene & No realiza & 2 & Colombiana \\
\hline Puerto Ezequiel & Microscopista & Sí & GG & 4 & Col/Per \\
\hline Puerto Huila & Microscopista & Sí, pero no exclusivo & GG & 5 & Col/Per \\
\hline Buenos Aires & Microscopista & Sí, pero no exclusivo & $\mathrm{GG}$ & 2 & Col/Per \\
\hline Tarapacá & Auxiliar en salud pública & Sí, pero no exclusivo & GG & 7 & Col/Per \\
\hline Tarapacá & Auxiliar o técnico en enfermería & Sí & GG & 8 & Col/Per \\
\hline Purísima Ventura & Auxiliar en salud pública & No tiene & No realiza & 4 & Col/Per \\
\hline Pupuña 1a Maloka & Microscopista & Sí & GG & 6 & Colombiana \\
\hline Arara & Microscopista & Sí, pero no exclusivo & GG & 4 & Brasileña \\
\hline Macedonia & Auxiliar en salud pública & No tiene & PDR & 5 & Colombiana \\
\hline San Pedro de Tipisca & Auxiliar en salud pública & No tiene & No realiza & 1 & Col/Per \\
\hline Doce de Octubre & Auxiliar en salud pública & No tiene & No realiza & 1 & Col/Per \\
\hline Puerto Nariño & Auxiliar en salud pública & Sí, pero no exclusivo & GG & 22 & Col/Per \\
\hline San Juan de Atacuary & Auxiliar en salud pública & No tiene & No realiza & 1 & Colombiana \\
\hline Siete de Agosto & Auxiliar en salud pública & No tiene & No realiza & 1 & Colombiana \\
\hline Naranjales & Auxiliar en salud pública & No tiene & No realiza & 2 & Col/Per \\
\hline $\begin{array}{l}\text { San Martín de } \\
\text { Amacayacu }\end{array}$ & Auxiliar en salud pública & No tiene & No realiza & 1 & Colombiana \\
\hline Santa Sofía & Auxiliar en salud pública & No tiene & No realiza & 5 & Colombiana \\
\hline
\end{tabular}


Anexo 2. Características sobre el diagnóstico de la malaria en los puntos visitados del departamento de Loreto (Perú), 2017.

\begin{tabular}{|c|c|c|c|c|c|}
\hline Puntos visitados & Tipo de recurso humano que labora & $\begin{array}{l}\text { Tienen microscopio } \\
\text { exclusivo para malaria }\end{array}$ & $\begin{array}{l}\text { Tipo de } \\
\text { diagnóstico que } \\
\text { realizan }\end{array}$ & $\begin{array}{l}\text { Número de } \\
\text { comunidades } \\
\text { que atienden }\end{array}$ & $\begin{array}{l}\text { Nacionalidad de } \\
\text { pacientes que } \\
\text { atienden }\end{array}$ \\
\hline Angusilla & Auxiliar o técnico en enfermería & No tiene & No realiza & 7 & Col/Per \\
\hline Nueva Esperanza & Auxiliar o técnico en enfermería & No tiene & PDR & 16 & Col/Per \\
\hline Bella Vista & Auxiliar o técnico en enfermería & No tiene & PDR & 4 & Peruana \\
\hline Santa Mercedes & Auxiliar o técnico en enfermería & Sí & GG & 6 & Col/Per \\
\hline San Antonio del Estrecho & Enfermera & Sí, pero no exclusivo & GG & 7 & Col/Per \\
\hline La Florida - 7 de Agosto & Auxiliar o técnico en enfermería & Sí & GG & 8 & Col/Per \\
\hline Remanso & Enfermera & Sí, pero no exclusivo & GG & 8 & Col/Per \\
\hline Huapapa & Auxiliar o técnico en enfermería & Sí, pero no exclusivo & GG & 5 & Col/Per \\
\hline El Álamo & Auxiliar o técnico en enfermería & Sí & GG & 5 & Col/Per \\
\hline Santa Rosa & Promotor en salud & Sí, pero no exclusivo & GG y PDR & 10 & Brasileña \\
\hline Vista Alegre & Promotor en salud & No tiene & PDR & 1 & Peruana \\
\hline San Antonio de Cocoa & Promotor en salud & No tiene & PDR & 15 & Col/Per \\
\hline Tierra Amarilla & Promotor en salud & No tiene & PDR & 2 & Col/Per \\
\hline Uranias & Promotor en salud & No tiene & PDR & 1 & Col/Per \\
\hline San Salvador & Promotor en salud & No tiene & No realiza & 1 & Peruana \\
\hline Puerto Alegre Pintadillo & Promotor en salud & No tiene & No realiza & 1 & Col/Per \\
\hline San Francisco de Yauma & Promotor en salud & No tiene & No realiza & 2 & Col/Per \\
\hline Puerto Alegría & Auxiliar o técnico en enfermería & Sí & GG y PDR & 10 & Col/Per \\
\hline
\end{tabular}

GG: gota gruesa; PDR: prueba de diagnóstico rápido; Col: colombiana; Per: peruana. 